Editorial: Hospital Metropolitano

ISSN (impreso) 1390-2989 - ISSN (electrónico) 2737-6303

Edición: Vol. 29 No 1 (2021) enero-marzo

DOl: https://doi.org/10.47464/MetroCiencia/vol29/1/2021/44-50

URL: https://revistametrociencia.com.ec/index.php/revista/article/view/134

Pág: $44-50$

\title{
Manejo de vía aérea difícil en secuencia de Pierre Robin. Reporte de un caso
}

\section{Difficult airway management in Pierre Robin Sequence. A case report}

\author{
Stefany Valeria Mendoza Navarrete' ${ }^{10}$; Mayra Viviana Villena Galarza ${ }^{2}$; \\ Xavier Raúl Mantilla Pinto ${ }^{2}$
}

\author{
Médico Residente de Postgrado de Anestesiología, Universidad San Francisco de Quito \\ Médico Anestesiólogo, Fellowship en Anestesiología Cardiotorácica, Hospital Metropolitano de Quito² \\ Recibido: 01/12/2020 Aceptado: 20/12/2020 Publicado: 29/01/2021
}

\begin{abstract}
RESUMEN
La secuencia de Pierre Robin (SPR) es una enfermedad rara, caracterizada por una tríada de malformaciones orofaciales como retrognatia, glosoptosis y fisura velopalatina media (paladar hendido); las cuales, generalmente provocan obstrucción de la vía aérea (OVA). La corrección de los defectos palatinos requiere en ocasiones varias intervenciones quirúrgicas, por esta razón el cuidado anestésico y abordaje de la vía aérea en forma adecuada, evita complicaciones asociadas a esta enfermedad. Se presenta el caso de un paciente de 1 año y 20 días de edad, con antecedentes de vía aérea difícil, intentos fallidos de intubaciones preliminares e intervenido quirúrgicamente para corrección de paladar hendido, luego de una técnica combinada de intubación nasal con fibrobroncoscopio, desplazamiento de glosoptosis con ayuda de pala número 2 de videolaringoscopio (Glidescope®); manejo transoperatorio anestésico y proceso de extubación con excelentes resultados. Destacamos la importancia de realizar una adecuada planificación multidisciplinaria prequirúrgica con valoración exhaustiva de la vía aérea (VA) por laringoscopía directa o fibrobroncospia flexible para conocer el sitio exacto de la obstrucción y evitar desenlaces adversos.
\end{abstract}

Palabras claves: Vía aérea difícil, paladar hendido, glosoptosis, obstrucción de la vía aérea.

\section{ABSTRACT}

Pierre Robin Sequence (PRS) is a rare disease characterized by a triad of orofacial malformations such as retrognathia, glossoptosis and velopalatine fissure (cleft palate). The malformations in PRS can lead into airway obstruction. The correction of the palatal defects sometimes requires several surgical interventions. Proper anesthetic care and a carefully planned approach to the airway can avoid complications associated with this disease. We present the case of a 1 year and 20 days old patient, with history of difficult airway, unsuccessful prior attempts of intubation, who underwent surgery to correct a cleft palate. We used a combined approach that included nasal intubation technique with a fiberoptic bronchoscope aided with a number 2 video laryngoscope blade (Glidescope $($ ) for displacement of the glossoptosys. Details of the intraoperative anesthetic management and subsequent successful extubation are provided. We highlight the importance of developing an adequate preoperative multidisciplinary plan of action, after a careful and detailed evaluation of the airway with direct laryngoscopy or fiberoptic bronchoscopy in order to identify the exact location of the obstruction and avoid adverse outcomes.

Keywords: Difficult airway, cleft palate, glossoptosis, airway obstruction.

Stefany Valeria Mendoza Navarrete: Mayra Viviana Villena Galarza: Xavier Raúl Mantilla Pinto:
IDs Orcid

https://orcid.org/0000-0001-8644-2181 https://orcid.org/0000-0002-0604-8721 https://orcid.org/0000-0003-4074-6811

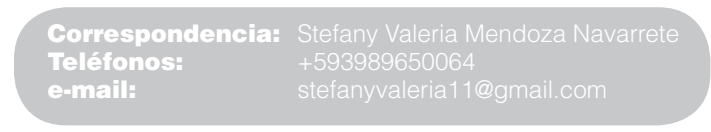




\section{INTRODUCCIÓN}

La secuencia de Pierre Robin (SPR) es una anomalía congénita del primer arco branquial, que es responsable de la formación de la mayoría de estructuras de la cara como la mandíbula, paladar, y oídos; es una enfermedad con herencia autosómica recesiva, presenta una prevalencia de 1 por cada 8.500 nacimientos $^{1}$; sin predilección de género, excepto en una variante ligada al cromosoma $X$.

Los primeros reportes se realizaron en 1845; sin embargo, Robin en 1923, definió como "secuencia" a la triada clásica de glosoptosis $(70 \%$ a $85 \%$ de pacientes); alteraciones mandibulares como hipognatia, micrognatia (91\%) o retrognatia, y deformidades palatinas (fisura velopalatina o paladar hendido en $10 \%$ a $15 \%)^{2}$; las cuales predisponen a obstrucción de la vía aérea (OVA) en cualquier momento de la vida. Estos hallazgos representan una heterogeneidad fenotípica, desencadenada por eventos mecánicos, genéticos y ambientales, que requieren un abordaje multidisciplinario para el manejo adecuado de la vía aérea (VA).

La presencia de hipoplasia mandibular causa prolapso de la base de la lengua hacia la pared posterior de la faringe que lleva a obstrucción de la vía aérea ${ }^{2-4}$. La hipoplasia mandibular conduce al fenotipo Robin ${ }^{3}$. Un 40\% de la SPR se expresa en forma aislada y $60 \%$ coexiste con otros síndromes con malformaciones craneofaciales ${ }^{1,5}$, afecciones neurológicas como síndrome de Stickler o síndrome de Nager ${ }^{6,7}$.

Otras manifestaciones que acompañan a este trastorno son base de nariz aplanada, dificultad para la respiración con obstrucción en la inspiración, déficit nutricional con falla de crecimiento, sindactilia o dedos hipoplásicos, glaucoma, microftalmia, malformaciones del pabellón auricular y anomalías en el esternón y costillas ${ }^{8,9}$.

En todos los casos de SPR es indispensable conocer el grado de obstrucción de la vía aérea (OVA) desde el nacimiento, para definir una terapéutica ideal. Ante una obstrucción supraglótica, por respuesta subóptima al procedimiento de adhesión de la lengua al labio o falla en la osteogénesis con distractores mandibulares, se recomienda realizar una traqueostomía de urgencia ${ }^{10,11,21}$.

Antes de cualquier intervención electiva para corrección de paladar hendido, es recomendable ejecutar una evaluación endoscópica exhaustiva de la VA, mediante nasoendoscopia y fibrobroncoscopia flexible. La laringoscopía directa y fibrobroncosco- pia permiten valorar apropiadamente estructuras de la VA, a menos que el paciente presente micrognatia severa, en el cual se recomienda realizar una maniobra de empuje mandibular bajo anestesia general ${ }^{3,21}$. A pesar de ello, no siempre se consigue la alineación completa de los ejes oral, faríngeo, laríngeo y tampoco está exenta de riesgos, al tratarse de un paciente con una VA en muchas ocasiones no intubable.

Du Plessis et al, en 2013 sugiere utilizar sondas nasofaríngeas que permitan corregir la OVA debido a la glosoptosis, problema importante en los recién nacidos con SPR ${ }^{12}$, por consiguiente, es necesario crear algoritmos que orienten a desarrollar un abordaje óptimo de la VA de manera segura y efectiva, con el fin de optimizar la eficiencia y la comunicación ${ }^{13,14}$.

\section{Caso clínico}

Paciente masculino de 1 año y 20 días de edad (Figuras $1 A$ y $1 B$ ), producto de segunda gestación, embarazo complicado por preeclampsia y sufrimiento fetal a las 35,4 semanas, con lo cual se indicó una cesárea de urgencia, sin incidentes. Durante el examen inicial se observaron dismorfias faciales y signos de dificultad respiratoria, ingresó a cuidados intensivos neonatales (UCIN) para recibir presión positiva continúa en la vía aérea (CPAP) por cuatro días e instauración de alimentación por sonda orogástrica (SOG) y luego a succión. A los 11 días de vida se confirmó sospecha diagnóstica de SPR mediante estudio genético y varios días después reinstala dificultad respiratoria, agrega problemas en la alimentación y apnea obstructiva del sueño con episodios de bradicardia; se logró estabilizar en forma transitoria con medidas de soporte como posición en decúbito prono alternando con decúbito lateral, oxígeno complementario por cánula nasal y sonda nasogástrica (SNG) para alimentación.

A los 40 días de vida se realizó el primer tiempo quirúrgico para distracción osteogénica mandibular y confección de gastrostomía para alimentación, previa intubación orotraqueal (IOT) a ciegas con tubo 3,5 sin balón, sin reporte de complicaciones (Figuras $2 A-2 F$ ). Al año de vida se programó nueva distracción osteogénica mandibular con palatoplastia (segundo tiempo quirúrgico) con dos intentos fallidos para abordar la vía aérea a pesar del uso de múltiples dispositivos, por lo cual se decidió diferir el procedimiento, para valoración complementaria de la vía aérea. (Figuras $3 A-3 C$ y 4 ). 


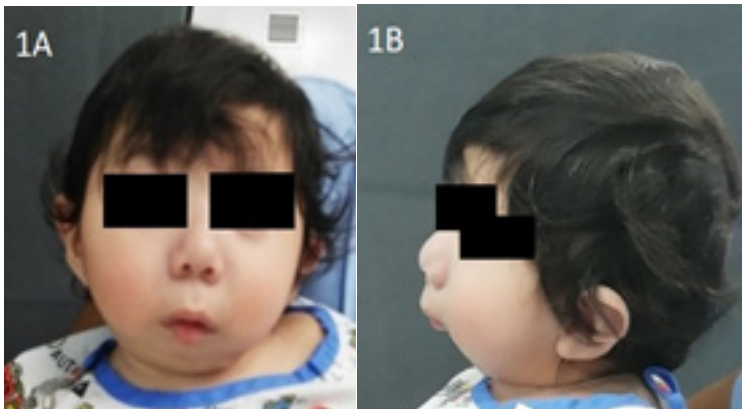

Figura 1. A) Vista frontal de fenotipo de paciente. B) Vista lateral de fenotipo de paciente.

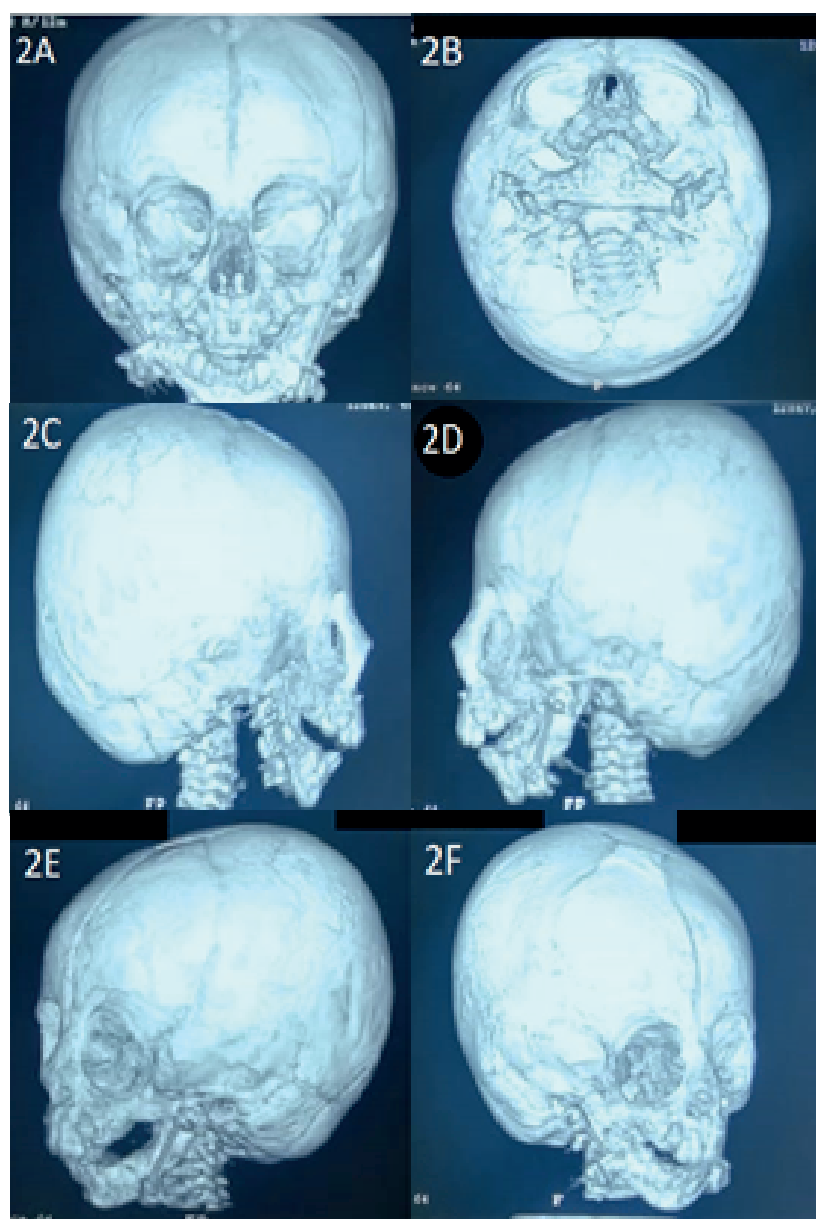

Figura 2. ( $A, B, C, D, E, F)$ Reconstrucción cráneofacial ósea con tomografía computarizada. Se observan distractores mandibulares de primera cirugía y micrognatia.

72 horas después, se vuelve a reprogramar la cirugía. El paciente ingresó a sala de operaciones con monitorización no invasiva según ASA y se cateterizó vía venosa periférica.

La inducción anestésica inhalatoria se realizó con Sevofluorano entre 4\% a 5\%, se administró 10 miligramos de lidocaína sin epinefrina (a 1,5 mg por kilogramo) por vía intravenosa (IV) y lidocaína tópica en la fosa nasal izquierda. La intubación nasotraqueal
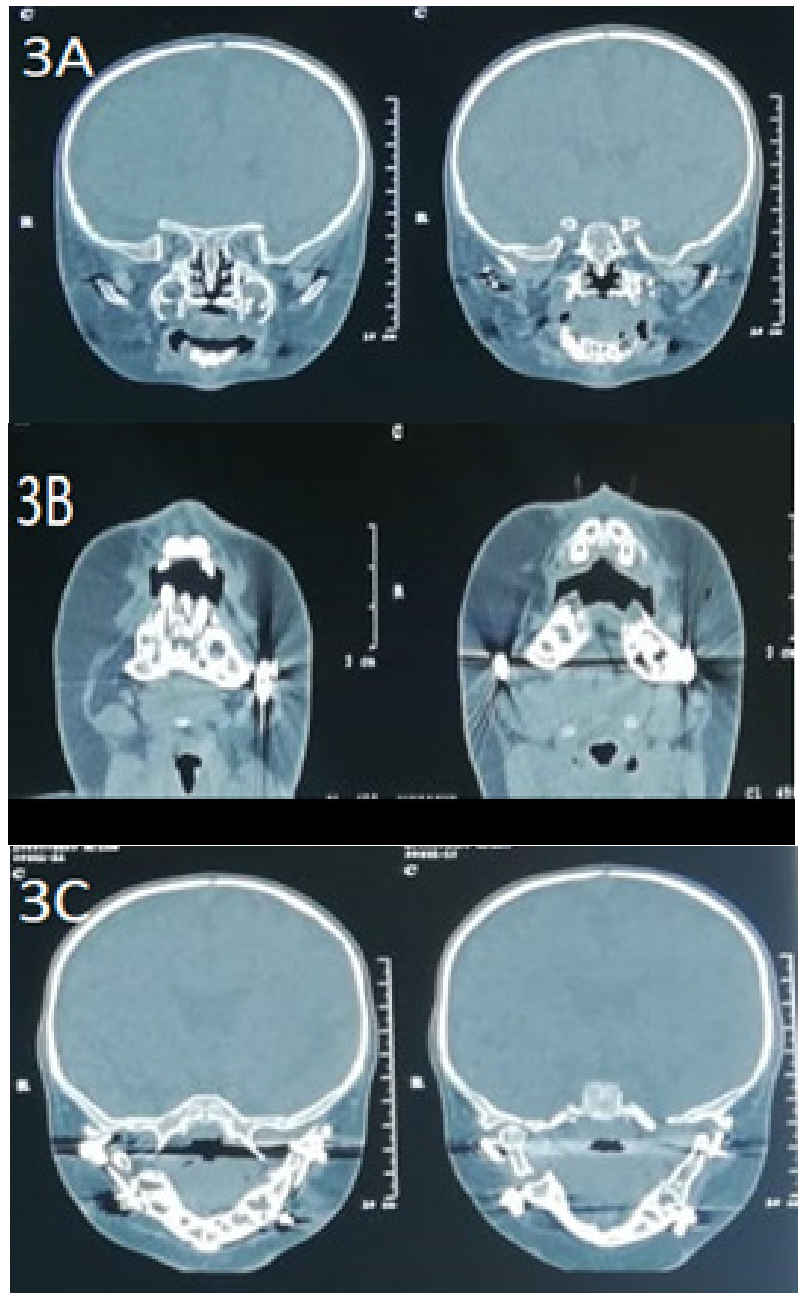

Figura 3. A) corte coronal. B) corte Axial. C) corte coronal. Se observan los defectos óseos palatinos y mandibulares.

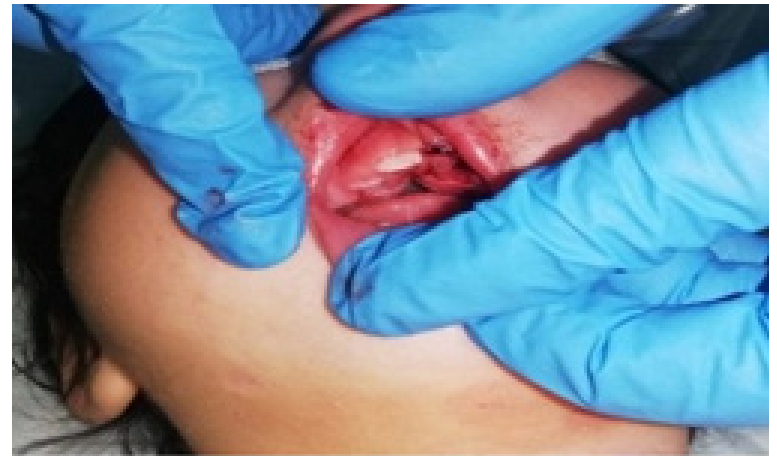

Figura 4. Apertura oral limitada y defecto congénito de paladar, previo a segunda cirugía.

(INT) se llevó a cabo con técnica mixta, fibrobroncoscopio nasal y con pala curva $\mathrm{N}^{\circ} 2$ de videolaringoscopio (Glidescope $\AA^{\circledR}$ ) en la cavidad oral (Figura 5). Por asimetría bucal y para evitar OVA con la base de la lengua que siempre se contacta con la pared posterior de la faringe, se usó la pala del videolaringoscopio, de esta forma se permitió el progreso con facilidad del fibrobroncoscopio hacia la hipofaringe (Figura 6 y $7 A-7 C$ ). 


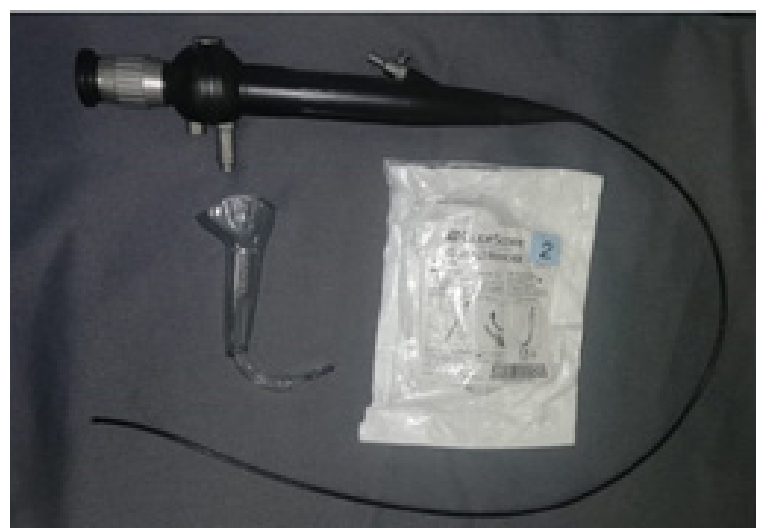

Figura 5. Dispositivos utilizados para manejo de vía aérea en paciente con síndrome de Pierre Robin.

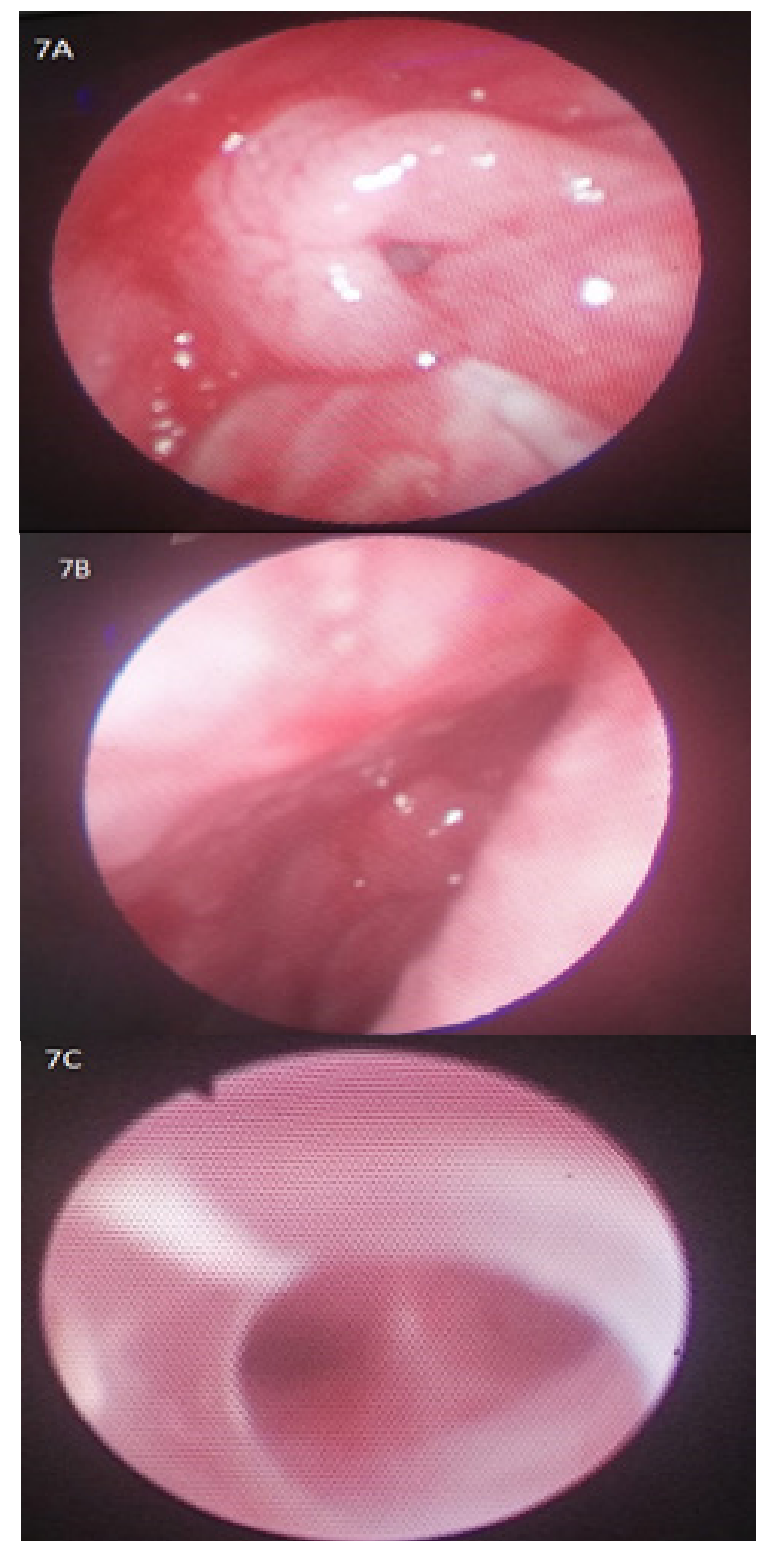

Figura 7. A) Vía aérea con espacio limitado por glosoptosia del paciente. B) Observación de epiglotis para inserción de tubo traqueal por fibrobroncoscopia nasal izquierda. C) Verificación de intubación adecuada al observarse la carina a través del tubo nasotraqueal.

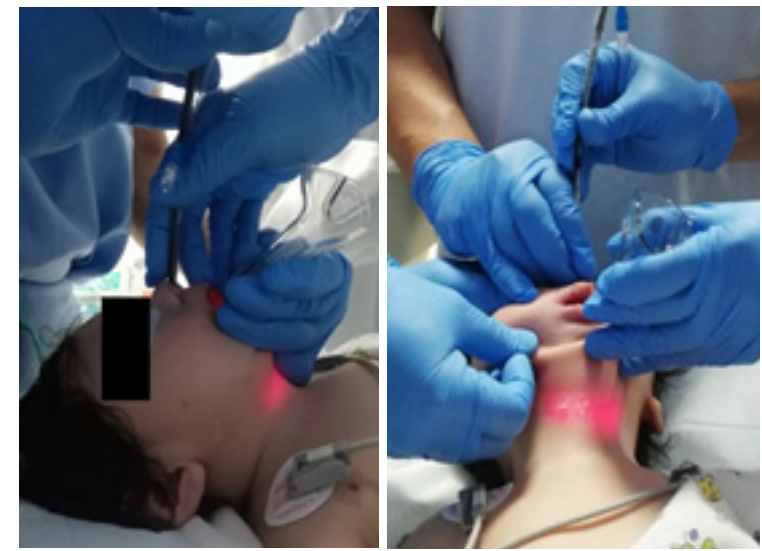

Figura 6. Vista lateral (izquierda) y frontal (derecha) del procedimiento de intubación mixta.

Se mantuvo una ventilación espontánea en el paciente, sin complicaciones. Se administró 10 miligramos de propofol $(1,5 \mathrm{mg} / \mathrm{kg})$ y 5 miligramos de rocuronio (0,6 mg/kg) por vía IV, y luego se aseguró la vía aérea.

El mantenimiento anestésico se logró con sevofluorano al $2 \%$, infusión IV continua de remifentanilo entre 0,15 a $0,30 \mathrm{mcg} / \mathrm{kg} / \mathrm{minuto}$ y dexmedetomidina $0.3 \mathrm{mcg} / \mathrm{kg} / \mathrm{hora}$. Adicionalmente se administró por vía IV 80 miligramos de ácido tranexánico $(10$ mg/ $\mathrm{kg}), 1,2$ miligramos de dexametasona $(0,15 \mathrm{mg} / \mathrm{kg})$, 4 miligramos de ketorolaco $(0,5 \mathrm{mg} / \mathrm{kg}), 230$ miligramos de cefazolina (30 mg/kg), 80 miligramos de paracetamol $(10 \mathrm{mg} / \mathrm{kg})$ y 1.2 miligramos de ondansetrón $(0,15 \mathrm{mg} / \mathrm{kg})$ en el intraoperatorio (Figuras 8 y 9).

El procedimiento quirúrgico de segunda distracción osteogénica mandibular con palatoplastia tuvo una duración de 6 horas y 20 minutos, sin incidentes anestésicos.

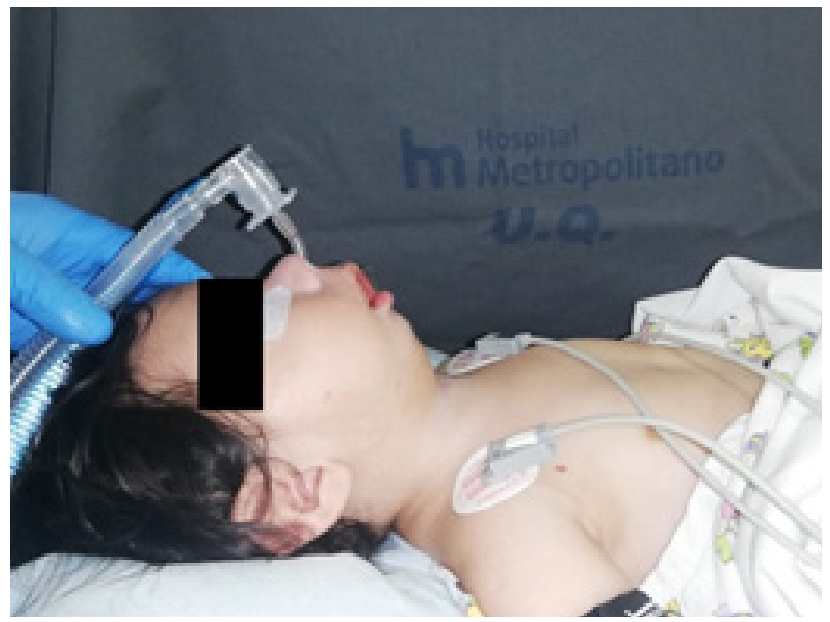

Figura 8. Intubación realizada con éxito mediante fibrobroncoscopía nasal modificada con uso de pala de videolaringoscopio N² vía oral para desplazamiento anterior de lengua. 


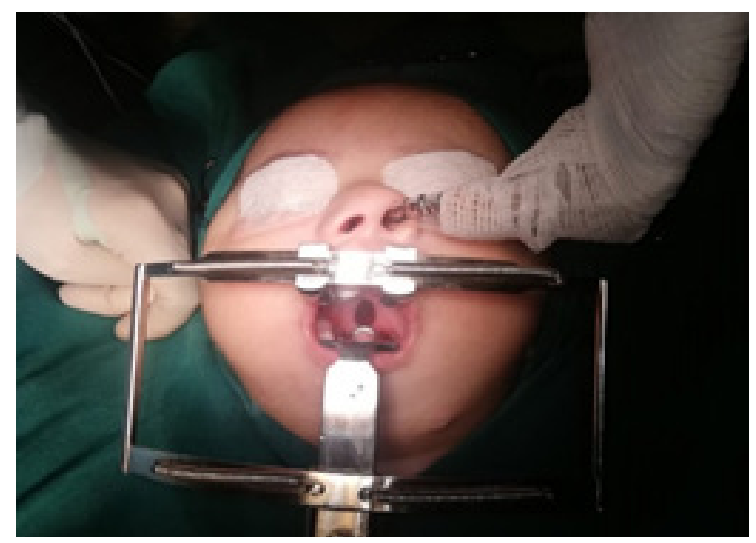

Figura 9. Visualización de defectos de la cavidad oral (paladar hendido) durante la intervención quirúrgica.

Se transporta de inmediato a la unidad de cuidados intensivos pediátricos (UCIP) con monitorización continua no invasiva, sedoanalgesia y soporte ventilatorio mecánico con INT con disminución progresiva de parámetros ventilatorios. A las 48 horas postquirúrgicas y con fibrobroncoscopia, se planificó extubación avanzada, sin complicaciones, con buena ventilación espontánea con mascarilla de oxígeno y sin OVA a pesar de edema facial (maxilar y mandibular) esperado de la cirugía.

\section{DISCUSIóN}

El abordaje de la VA en los pacientes con SPR es considerada como un desafío importante en la práctica anestesiológica, justificado por las malformaciones faciales que presentan y la alta probabilidad de morbimortalidad asociada al deterioro respiratorio y nutricional que desarrollan estos pacientes a lo largo de su vida, si no se corrigen. La asociación de glosoptosis con hipoplasia mandibular conlleva a reducción del área de la cavidad oral, con difícil apertura bucal (Figura 9), resultando en un riesgo de OVA ${ }^{15}$.

Como consecuencia, pueden ocasionarse episodios de hipoxemia y requerir en algún momento un manejo avanzado de la VA; inclusive, puede llegar a ser una condición mortal por la mala visualización por laringoscopia, al transformarse en una VA no ventilable y no intubable.

Es evidente que los pacientes con SPR tienen diferentes grados de afectación en cuanto a presentación, severidad y grado de OVA. De todo esto dependerá el tratamiento médico a recibir, sea en forma conservadora o quirúrgica. En el manejo conservador se propone con el posicionamiento en decúbito lateral o decúbito prono al dormir, uso de cánulas nasofaríngeas, CPAP o intubación endotraqueal ${ }^{12}$. En el manejo quirúrgico (invasivo) se indica glosopexia, traqueostomía y distracción osteogénica mandibular, según el grado de severidad.
Todos los procedimientos que requieren de anestesia general e intubación orotraqueal o nasotraqueal, pueden generar dificultades o complicaciones al momento de abordar la VA, inclusive con personal médico experimentado ${ }^{16}$. El manejo multidisciplinario, planificado, basado en protocolos de manejo de VA difícil, además de experiencia y destrezas en el manejo de la fibrobroncoscopia, permitió al paciente mantenerse en un plano anestésico adecuado con mantenimiento de la ventilación espontánea. Antes de llevar a cabo cualquier procedimiento quirúrgico, es esencial una evaluación expedita de la VA, mediante laringoscopía y fibrobroncoscopía diagnóstica y sobre todo en los pacientes con sospecha de VA difícil, fundamentada en los antecedentes personales, factores de riesgo, comorbilidades asociadas e historia previa de intubaciones fallidas; motivos por los cuales pueden llevar a dificultades en el abordaje inicial, como sucedió en nuestro caso.

De esta manera se evita la ansiedad generada por el procedimiento, tanto en familiares como en el personal de salud, se disminuyen gastos y la probabilidad de incidentes mayores asociados a intubaciones fallidas como hipoxemia, laringoespasmo, paro cardiorrespiratorio e incluso deceso ${ }^{17}$.

En nuestro caso, la laringoscopia directa no se pudo realizar en forma adecuada, puesto que la presencia de los distractores osteogénicos mandibulares fueron la limitante más importante (Figura 4), los cuales ocasionaron fallas e imposibilidad de la intubación endotraqueal y por lo tanto dificultad en un manejo apropiado de la VA.

Adicionalmente, por el fenotipo del paciente se pudo comprobar asimetría de crecimiento del macizo facial y la mandíbula, con un marcado estancamiento de la mandíbula a pesar de la distracción mandibular previa. Por los antecedentes de intubación fallida (con laringoscopio convencional, Mc Coy, videolaringoscopios CMAC $₫$ y Ambuscope $®$ ) y la enfermedad de base de nuestro caso, se planteó tomar la conducta más idónea para manejo de la VA, a través de un equipo multidisciplinario conformado por anestesiólogos experimentados, otorrinolaringólogo y cirujano plástico; con objetivos definidos a cumplirse como una INT planificada oportunamente bajo inducción inhalatoria, y mantener al paciente en un plano anestésico que le permita conservar su ventilación espontánea ${ }^{18}$, a fin de proteger la VA y evitar incidentes que podrían cambiar la conducta elegida, como por ejemplo la realización de procedimientos invasivos de urgencia (traqueostomía).

Mediante el consenso del equipo multidisciplinario, se realizó con éxito una intubación mixta por medio de dos dispositivos de manejo avanzado de VA diff́$\mathrm{Cil}^{18}$ (fibrobroncoscopio flexible por vía nasal y pala del videolaringoscopio por vía oral), tal como se observa en la figura 5. 
En toda VA difícil, el anestesiólogo se enfrenta a varios retos tanto en la intubación como en la extubación. La dificultad en la intubación acompañada de ventilación difícil, son factores de riesgo para extubación fallida ${ }^{19,20}$; por lo cual se indica una fibrobroncoscopia previamente a la extubación, tal como se programó en nuestro caso; de esta manera se evitó mantener al paciente con una ventilación mecánica prolongada, a veces innecesaria; disminuyendo e inclusive evitando complicaciones asociadas de tipo neurológicas por sedoanalgesia (en la neuroplasticidad), infecciosas (neumonía del ventilador) y propias de la cirugía (ayuno prolongado e incapacidad de realización de actividades diarias).

\section{CONCLUSIONES}

Destacamos la importancia de la evaluación y revaloración de la vía aérea para cada procedimiento quirúrgico programado en pacientes con secuencia de Pierre Robin, puesto que a pesar de que conozcamos al paciente, siempre es necesaria la revaloración exhaustiva ya que esta patología tiene tendencia a acrecentar su asimetría facial, y esto es el primer punto clave para evitar errores durante la manipulación de la vía aérea. El segundo, es el trabajo en equipo y la comunicación adecuada en todo el procedimiento, lo cual fue relevante para el desarrollo exitoso del proceso de intubación, donde se tenía prevista una VA difícil y el antecedente de múltiples intentos de intubación fallida con diversos dispositivos avanzados de manejo de VA. Por último, fue la combinación de la fibrobroncoscopía nasal como un instrumento útil para valorar la anatomía retrofaríngea y el uso de la pala del videolaringoscopio como auxiliar, que ayudó a superar la obstrucción que ocasionaba la lengua durante el proceso de intubación.

\section{CONFLICTOS DE INTERÉS}

Los autores declaran no tener conflictos de interés de ningún tipo.

\section{CONTRIBUCIÓN DE LOS AUTORES EN LA INVESTIGACIÓN DE TRABAJO PARA EL CASO CLÍnico}
a. Concepción y diseño del trabajo: MN, VG.
b. Recolección/obtención de resultados: MN, VG.
c. Análisis e interpretación de datos: MN, VG, MP.
d. Redacción del manuscrito: MN, VG.
e. Revisión crítica del manuscrito: MP.
f. Aprobación de su versión final: MP.
g. Aporte de pacientes o material de estudio: MN, VG, MP.

h. Obtención de financiamiento: no aplica.

i. Asesoría estadística: MN, VG, MP.

j. Asesoría técnica o administrativa: MN, VG, MP.

k. Otras contribuciones búsqueda bibliográfica: MN, VG, MP.

\section{FINANCIAMIENTO}

Por medios propios

\section{AGRADECIMIENTOS}

Agradecemos al equipo de anestesiólogos del Hospital Metropolitano de Quito quienes intervinieron en el procedimiento quirúrgico y en el abordaje de la vía aérea y nos proporcionaros las imágenes para el desarrollo de esta publicación, especialmente al Dr. Gustavo Cifuentes, Dr. Cristian Pozo, Dr. Vinicio Garzón, y Dr. Francisco Altamirano, y al equipo de otorrinolaringólogos, Dr. Jorge Cañar y Dr. Rodrigo Castrillón.

\section{REFERENCIAS BIBLIOGRÁFICAS}

1. Marston AP, Lander TA, Tibesar RJ, Sidman JD. Airway management for intubation in newborns with Pierre Robin sequence. Laryngoscope 2012; 122(6):1401-4. PubMed

2. Robin P. Glossoptosis due to atresia and hypotrophy of the mandible. Am J Dis Child. 1934;48(3):541-547. doi:10.1001/archpedi.1934.01960160063005

3. Evans et al. Robin Sequence: From Diagnosis to Development of an Effective Management Plan. PEDIATRICS. 2011; 127(5): 936-948. doi:10.1542/peds.2010-2615

4. Hsieh S., Woo A. Pierre Robin Sequence. Clin Plastic Surg. 2019;46(2): 249-159. https://doi.org/10.1016/j.cps.2018.11.010

5. Sevilla-Paz Soldan RM et al. Síndrome de Pierre Robin: reporte de un caso. Rev Méd-Cient "Luz Vida”. 2013;4(1) ISSN 2219-8032 :58-62. Disponible en: https://www.redalyc.org/articulo.oa?id=325029251012

6. Suárez Barrientos et al. Síndrome de Pierre Robin. Gaceta Médica Boliviana 2010; 33 (1): 38-43. Disponible en: http://www.scielo.org.bo/ $\mathrm{pdf} / \mathrm{gmb} / \mathrm{v3} 3 \mathrm{n} 1 / \mathrm{a} 08 . \mathrm{pdf}$

7. Tiol-Carrillo A. Secuencia malformativa de Pierre Robin: informe de un caso y revisión de la literatura. Revista ADM 2017; 74 (3): 146-151. Disponible en: https://www.medigraphic.com/pdfs/adm/od-2017/ od173g.pdf

8. López Salgado ML, Reyes Castañeda EG. "Distracción mandibular como tratamiento en secuencia de Robin. Caso clínico." Rev Mex Cir Buc y Max 2012; 8(3): 84-91. Disponible en: https://www.medigraphic.com/pdfs/cirugiabucal/cb-2012/cb123b.pdf

9. Llanio Navarro S et al. Síndromes. Primera Edición. La Habana: Editorial Ciencias Médicas; 2005

10. Paes EC et al. A pragmatic approach to infants with Robin sequence: a retrospective cohort study and presence of a treatment algorithm. Clin Oral Invest 2015; 19:2101-2114 DOI 10.1007/s00784-015-1407-6

11. Ching Jessica et al. A Simple Mandibular Distraction Protocol to Avoid Tracheostomy in Patients with Pierre Robin Sequence. The Cleft Palate-Craniofacial Journal 2017; 54(2):000-000. https://doi. org/10.1597/14-211

12. M. du Plessis S. et al. Airway and feeding problems in infants with Fairbairn-Robin triad deformities. Curationis. 2013; 36(1): 1-9. Disponible en: http://www.scielo.org.za/pdf/cura/v36n1/23.pdf

13. Hicks et al. An Algorithm for Airway Management in Patients With Pierre Robin Sequence. The Journal of Craniofacial Surgery. 2018; 0(0): 1-6. DOI: 10.1097/SCS.0000000000004489.

14. Collins et al. Airway Management in Pierre Robin Sequence: Patterns of Practice. The Cleft Palate-Craniofacial Journal. 2014; 51(3) pp. 283-289. DOI: 10.1597/12-214 
15. Guerrero Domínguez $R$ et al. Manejo de la vía aérea en un paciente con secuencia de Pierre Robin para la cirugía de distracción osteogénica mandibular. Rev Elect Anestesiar. 2015; 7(8) :1. Disponible en: http://revistaanestesiar.org/index.php/rear/article/view/182/317

16. Amerigo Giudice et al. Pierre Robin Sequence: A comprehensive narrative review of the literature over time. Journal of Stomatology Oral and Maxilofacial Surgery. 2018. Pp 1-33. DOI: https://doi.org/ doi:10.1016/j.jormas.2018.05.002

17. Chang AB, Masters I, Williams GR, et al. A Modified Nasopharyngeal Tube to Relieve High Upper Airway Obstruction. Pediatr Pulmonol 2000; 29: 299-306.

18. Andreu E, Schmucker E, Drudis R, Farré M, Franco T, Monclús E, et al. Algorithm for pediatric difficult airway. Rev Esp Anestesiol Reanim. 2011; 58:304-11. PubMed
19. Pérez-González JA, García-Cartaya Z. Síndrome de Pierre Robin. Presentación de un caso clínico. Panorama Cuba y Salud. 2011; 6 (1): 44 46. Disponible en: https://www.redalyc.org/pdf/4773/477348944008. pdf

20. Sorbello M, Frova G. When the end is really the end? The extubation in the difficult airway patient. Minerva Anestesiol. 2013; 79(2):194-9. Disponible en: https://pubmed.ncbi.nIm.nih.gov/23090106/

21. Resnick, Cory M. et al. Early Management of Infants With Robin Sequence: An International Survey and Algorithm. Journal of Oral and Maxillofacial Surgery, Volume 77, Issue 1, 136-156. DOI: https://doi. org/10.1016/j.joms.2018.05.031 